\title{
Résumé de recherches sur la symétrie des polyèdres non eulériens.
}

(Par M. Camille Jordan à Châlon s. Saône.)

Une surface sera dite d'espèce $(m, n)$ si elle est limitée par $m$ contours fermés et si l'on peut d'autre part y tracer $n$ contours fermés ne se coupant eux - mêmes ni mutuellement, sans la partager en deux régions distinctes.

L'importance des deux paramètres $m$ et $n$ ressort des propositions suivantes:

$1^{\circ}$. Une surface d'espèce $(m, n)$ est $m+2 n$ fois continue (zusammenhängend), en donnant à ce terme la même définition que M. Riemann (tome 54 de ce Journal). On doit excepter le cas où $m=0$ : la surface est alors non plus $2 n$ fois, mais $2 n+1$ fois continue.

$\boldsymbol{2}^{\circ}$. Tout contour tracé sur une surface d'espèce $(m, n)$ peut être réduit par une déformation progressive à une combinaison de certains contours simples, en nombre $m+2 n$.

$3^{\circ}$. Pour que deux surfaces flexibles et extensibles à volonté soient applicables l'une sur l'autre, il faut et il suffit qu'elles soient de même espèce.

$4^{\circ}$. On a dans toute surface polyédrique d'espèce $(m, n)$ entre le nombre $\boldsymbol{F}$ des faces, celui $\boldsymbol{S}$ des sommets et celui $\boldsymbol{A}$ des arêtes, la relation

$$
F+S=A+2-m-2 n
$$

qui n'est autre que le théorème d'Euler généralisé.

En posant $m=0$ et faisant varier $n$, on aura les diverses espèces de polyèdres fermés.

Les polyèdres de l'espèce $(0,0)$ ne sont autres que ceux que j'ai appelés eulériens dans le mémoire précédent. Le problème de la symétrie se pose d'une manière analogue dans les autres espèces de polyèdres: mais les résultats obtenus sont essentiellement différents d'une espèce à l'autre.

Prenons par exemple les polyèdres de l'espèce $(0,1)$. (Un polyèdre présentant l'aspect général d'un tore appartiendrait à cette espèce). Il résulte de mon analyse que ces polyèdres peuvent offrir trois sortes différentes de symétrie. 
1 $^{\circ}$ Symétrie $m n$-quaternaire. Polyèdres offrant deux systèmes distincts de $m n$ éléments à rotation quaternaire et un système de $2 m n$ éléments à rotation binaire (ou d'arêtes à retournement). Les autres éléments et arêtes sont $4 m n$ fois répétés. Les entiers $m$ et $n$ peuvent être quelconques, sauf cette restriction que si l'un d'eux se réduit à l'unité, l'autre doit se réduire à 1 , ou à 2 .

$2^{\circ}$. Symétrie $m n$-binaire. Polyèdres offrant quatre systèmes distincts de $m n$ éléments à rotation binaire, (chacun de ces systèmes pouvant être remplacé par un système d'arêtes à retournement). Les autres éléments et arètes sont $2 m n$ fois répétés. Les entiers $m$ et $n$ sont absolument quelconques.

$3^{\circ}$. Symétrie $m n$-aire. Polyèdres présentant $m n$ aspects semblables: chaque élément ou arête étant $m n$ fois répété. Les entiers $m$ et $n$ sont quelconques.

Dans les trois cas ci-dessus, les entiers $m$ et $n$ pouvant être pris aussi grands que l'on veut, on peut toujours construire un polyèdre de l'espèce $(0,1)$ qui soit pareil à lui-même sous un nombre d'aspects qui dépasse toute limite assignée a priori. La même circonstance se présentait pour les polyèdres eulériens, qui sont susceptibles d'offrir une symétrie de rotation dont l'ordre reste arbitraire. Mais il est digne de remarque que les polyèdres appartenant à ces deux espèces sont les seuls polyèdres fermés qui jouissent de cette propriété: cela résulte de la proposition suivante:

Théorème. Le nombre $K$ des aspects sous lesquels un polyèdre d'espèce $(0, n)$ peut être pareil à lui-même est nécessairement limité, si $n>1$.

La démonstration de cette proposition générale est assez délicate: en voici l'abrégé.

On peut supposer qu'aucune face du polyèdre considéré n'est sa propre homologue sous plusieurs aspects différents: car si cela avait lieu, on pourrait prendre un point dans l'intérieur de la face et le joindre aux divers sommets de son contour, de manière à remplacer la face par un système de facettes triangulaires: en opérant de même sur les faces semblables, on aura un nouveau polyèdre d'espèce $(0, n)$ jouissant des mêmes symétries, et où aucune face ne sera sa propre homologue: on pourra étudier ce polyèdre au lieu du primitif.

Cela posé, soient $f$ une face, $f_{1}, f_{2}, \ldots$ les faces pareilles: s'il en existe d'autres, l'une d'elles, $g$, sera contigue suivant une arête à l'une des précédentes, à $f$ par exemple: ses homologues $g_{1}, g_{2}, \ldots$ sont respectivement 
contigues à $f_{1}, f_{2}$ etc. .... Si le polyèdre contient d'autres faces, l'une d'elles $h$ est contigue suivant une arête à quelqu'une des précédentes, à $g$ par exemple: ses homologues $h_{1}, h_{2} \ldots$ seront respectivement contigues à $g_{1}$, $g_{2} \ldots$ etc. On peut épuiser ainsi toutes les faces du polyèdre: alors les faces $f, g, h \ldots$ etc. d'une part, $f_{1}, g_{1}, h_{1} \ldots$ etc., $f_{2}, g_{2}, h_{2} \ldots$ etc. d'autre part, forment des régions $R, R_{1}, R_{2} \ldots$ pareilles entre elles, en nombre $K$, et qui par leur réunion constituent le polyèdre.

Supposons que chacune de ces régions soit de l'espèce $(\mu, \nu)$. Soient $F, S, A$ les nombres des faces, sommets et arêtes de chacune d'elles: on a:

$$
F+S=A+2-\mu-2 \nu
$$

ou en ajoutant les résultats relatifs aux $K$ faces

$$
\text { (1.) } \quad K F+K S=K A+(2-\mu-2 \nu) K \text {. }
$$

Soient $F^{\prime}, S^{\prime}, A^{\prime}$ les nombres des faces, sommets et arêtes du polyèdre: on a $F^{\prime}=K F$. D'autre part toute arête contenue sur le contour d'une région se trouve sur celui d'une autre région: elle est par suite comptée deux fois dans $K A$ : on a donc en désignant par $\alpha$ le nombre des arêtes situées sur le contour de $R$

$$
\boldsymbol{K} \boldsymbol{A}=\boldsymbol{A}^{\prime}+\frac{1}{2} \alpha \boldsymbol{K} \text {. }
$$

Enfin les sommets situés sur le contour de $\boldsymbol{R}$ sont tous situés sur le contour de deux ou d'un plus grand nombre de régions: soit en général $\beta_{\lambda}$ le nombre de ceux qui sont situés sur $\lambda$ régions: on aura:

$$
K S=S^{\prime}+\left(\frac{1}{2} \beta_{2}+\frac{2}{3} \beta_{3}+\cdots+\frac{\lambda-1}{\lambda} \beta_{\lambda}\right) K .
$$

Substituant ces valeurs dans (1.) il vient

$$
\left(2-\mu-2 \nu+\frac{1}{2} \alpha-\frac{1}{2} \beta_{2}-\frac{2}{3} \beta_{3}-\cdots-\frac{\lambda-1}{\lambda} \beta_{\lambda} \ldots\right) K=F^{\prime}+S^{\prime}-A^{\prime}=2-2 n \text {. }
$$

Dans chacun des contours qui bordent $R$ le nombre des arêtes est égal au nombre des sommets: on aurait donc en additionnant les résultats relatifs à ces divers contours

$$
\alpha=\beta_{2}+\beta_{3}+\cdots+\beta_{\lambda}+\cdots
$$

si ces contours ne se touchaient nulle part: mais si $\gamma_{2}$ sommets appartiennent à deux des contours limites, $\gamma_{3}$ à trois d'entre eux etc. ... on aura plus généralement

$$
\alpha=\beta_{2}+\beta_{3}+\cdots+\beta_{\lambda}+\cdots+\gamma_{2}+2 \gamma_{3}+\cdots+(s-1) \gamma_{s} .
$$


D'autre part les diverses faces de $\boldsymbol{R}$ étant contigues entre elles suivant des arêtes, on peut passer d'un point quelconque de $R$ à un autre sans toucher les contours limites. Si donc on suppose la continuité de $\boldsymbol{R}$ détruite aux sommets où les contours se rejoignent, ce qui demandera $\gamma_{2}+2 \gamma_{3}+\cdots+(s-1) \gamma_{s} \cdots$ transversales infiniment petites, $R$ restera encore d'une seule pièce: d'où la relation

$$
\mu+2 \nu \equiv \gamma_{2}+2 \gamma_{3}+\cdots+(s-1) \gamma_{s}+\cdots+1
$$

Posons

$$
\mu+2 \nu-\gamma_{2}-2 \gamma_{3}-\cdots-(s-1) \gamma_{s}-1=\varphi:
$$

l'équation (2.) deviendra

$$
\left\{-1+\varphi+\frac{1}{2} \gamma_{2}+\cdots+\frac{s-1}{2} \gamma_{s}+\cdots+\frac{1}{6} \beta_{3}+\cdots+\frac{\lambda-2}{2 \lambda} \beta_{\lambda}\right\} K=2 n-2 .
$$

Cela posé, $\varphi+\frac{1}{2} \gamma_{2}+\cdots+\frac{s-1}{2} \gamma_{s}=\frac{N}{2}, N$ étant un entier positif ou nul.

Si $N>2$ le multiplicateur de $K$ est $\overline{>}_{\frac{1}{2}}$ : donc la valeur de $K$ donnée par l'équation (3.) ne peut dépasser $2(2 n-2)$.

Si $N=2$, le multiplicateur de $K$ est égal à

$$
\frac{1}{6} \beta_{3}+\cdots+\frac{\lambda-2}{2 \lambda} \beta_{\lambda} \cdots \equiv \frac{1}{6}\left(\beta_{3}+\cdots+\beta_{\lambda}\right) \equiv \frac{1}{6} ;
$$

car on ne peut avoir à la fois $\beta_{3}=0, \ldots \beta_{\lambda}=0$, ce qui donnerait $2 n-2=0$ ou $n=1$, cas rejeté de nos hypothèses. On aura donc $K \equiv 6(2 n-2)$.

Enfin si $N=1$, ou $=0$, l'équation (3.) donnera

$$
\left(\frac{N-2+\beta_{3}+\cdots+\beta_{\lambda}}{2}-\frac{1}{3} \beta_{3}-\cdots-\frac{1}{\lambda} \beta_{\lambda} \ldots\right) K=2 n-2 .
$$

D'ailleurs le multiplicateur de $K$ est $\sum \frac{N-2}{2}+\frac{\beta_{3}+\cdots+\beta_{\lambda}}{6}$; d'autre part, pour que $K$ soit entier et $>1$, il faut que ce multiplicateur ne dépasse pas $n-1$ : on a donc $\beta_{3}+\cdots+\beta_{\lambda} \ldots<6 n-3 N+1$, limite que nous désignerons par $M$.

Or soit donné en général un système de solutions entières de l'équation

$$
\left(\frac{a}{b}-\frac{1}{3} \beta_{3}-\cdots-\frac{1}{\lambda} \beta_{\lambda} \ldots\right) K=L
$$

jointe à l'inégalitẻ

$$
\beta_{3}+\cdots+\beta_{\lambda} \cdots<M,
$$

et désignons par $\beta_{\lambda_{1}}, \beta_{\lambda_{2}} \ldots$ celles des inconnues $\beta$ qui ne sont pas nulles: on peut assigner une limite supérieure à chacun des indices $\lambda_{1}, \lambda_{2} \ldots$ 
Car soit $\lambda_{1}$ le plus petit de ces indices: posons $\frac{1}{\lambda_{1}} \beta_{1}+\frac{1}{\lambda_{2}} \beta_{2}+\cdots=\frac{p}{q}$, cette fraction étant supposée réduite à sa plus simple expression, ainsi que $\frac{a}{b}$ : On aura $\left(\frac{a}{b}-\frac{p}{q}\right) K=L$, d'où $K=\frac{L b q}{a q-b p}=\frac{L b e}{a e-d p}$, en posant $b=d \delta, q=e \delta$, $\delta$ étant le plus grand commun diviseur de $b$ et de $q$.

$K$ étant entier, $a e-d p$ divise $L b e$ : mais il est premier à $e$ : donc il divise $L b$ : on a donc $a e-d p \equiv L b$ ou en multipliant par $\delta$,

$$
a q-b p \equiv L b \delta \equiv L b^{2} . \quad \text { Mais } \quad \frac{p}{q} \equiv \frac{\beta_{\lambda_{1}}+\beta_{\lambda_{2}}+\cdots}{\lambda_{1}}<\frac{M}{\lambda_{1}}
$$

d'où $q>\frac{\lambda_{1}}{M} p:$ donc enfin

$$
\left(\frac{a \lambda_{1}}{M}-b\right) p \equiv L b^{2}
$$

d'où $\lambda_{1}<\frac{M}{a}\left\{\frac{L b^{2}}{p}+b\right\}<\frac{M}{a}\left(L b^{2}+b\right)$.

La quantité $\lambda_{1}$ étant ainsi limitée, de même que $\beta_{\lambda_{1}}$ qui est au plus égal à $M, \frac{1}{\lambda_{1}} \beta_{\lambda_{1}}$ ne sera susceptible que d'un nombre fini de valeurs: prenons l'une d'elles à volonté et posons $\frac{a}{b}-\frac{1}{\lambda_{1}} \beta_{\lambda_{1}}=\frac{a^{\prime}}{b^{\prime}}$ : on aura

$$
\left(\frac{a^{\prime}}{b^{\prime}}-\frac{1}{\lambda_{2}} \beta_{\lambda_{2}}-\frac{1}{\lambda_{3}} \beta_{\lambda_{3}} \ldots\right) K=L
$$

avec

$$
\beta_{\lambda_{2}}+\beta_{\lambda_{3}}+\cdots<M-\beta_{\lambda_{1}}
$$

on en conclut comme tout à l'heure l'inégalité

$$
\lambda_{2}<\frac{M-\beta_{\lambda_{1}}}{a^{\prime}}\left(L b^{\prime 2}+b^{\prime}\right)
$$

$\lambda_{2}$ est ainsi limité, de même que $\beta_{\lambda_{2}}$ : on reconnaît de même que $\lambda_{3}$ etc. sont limités.

Tous les entiers $\lambda_{1}, \lambda_{2}, \lambda_{3} \ldots \beta_{\lambda_{1}}, \beta_{\lambda_{2}}, \beta_{\lambda_{3}} \ldots$ étant ainsi renfermés dans certaines limites, le multiplicateur de $K, \frac{a}{b}-\frac{1}{\lambda_{1}} \beta_{\lambda_{1}}-\frac{1}{\lambda_{2}} \beta_{\lambda_{2}} \ldots$ n'est susceptible que d'un nombre limité de valeurs: en les substistuant successivement dans l'équation (4.), on aura les valeurs correspondantes de $K$, en nombre limité.

La considération des aspects peut s'étendre aux surfaces polyédriques non fermées: soit $\boldsymbol{P}$ une semblable surface, d'espèce $(m, n)$ : ajoutons $-\mathrm{y} \boldsymbol{m}$ faces 
auxiliaires ayant respectivement pour contour les $m$ contours limites de $P$ : on aura une nouvelle surface $Q$ d'espèce $(0, n)$ : et si $P$ est pareil à luimême sous plusieurs aspects, $Q$ le sera sous ces mêmes aspects : et de plus les $m$ faces auxiliaires seront leurs propres homologues sous tous ces aspects.

Si l'on veut que $P$ soit pareil à lui-même sous un nombre d'aspects illimité, $K$, il en sera de même de $Q$ : donc $n<2$ comme on vient de le voir. D'ailleurs on ne peut avoir $n=1$, car dans ce cas aucun élément de $Q$ ne pouvant être doué d'une rotation plus que quaternaire, les $m$ faces auxiliaires ne peuvent être leurs propres homologues sous plus de $4 \mathrm{~m}$ aspects: d'où $K \equiv 4 m$. Enfin si $n=0$, comme tous les éléments de $Q$, excepté un, ou deux au plus, ne peuvent être leurs propres homologues que sous un nombre limité d'aspects, cinq au plus, on ne peut avoir $m>2$ : car si $m=3$ l'une au moins des trois faces auxiliaires ne pourrait être son homologne sous plus de cinq aspects et par suite aurait au moins $\frac{1}{5} K$ homologues distinctes relativement aux $K$ aspects considérés. On aurait donc $m \equiv \frac{1}{5} K$, d'où $K \equiv 5 m ; K$ serait donc limité.

Ainsi les seules surfaces polyédriques qui puissent être pareilles à elles-mêmes sous un nombre illimité d'aspects sont (avec celles des espèces $(0,0)$ et $(0,1)$ déjà trouvées) celles des espèces $(1,0)$ et $(2,0)$.

Châlon sur Saône 1866. 